most likely represent a cluster of cases in the SWSLHD area but may alternatively reflect either better recognition or increased incidence of CJD.

\section{PROSPECTIVE STUDY OF 24-HOUR LEVODOPA- CARBIDOPA INTESTINAL GEL THERAPY FOR UNRESPONSIVE FREEZING OF GAIT}

Sai Nagaratnam*, Florence Ching-Fen Chang, David S Tsui, Neil Mahant, Nigel Wolfe, Samuel D Kim, Ainhi D Ha, Jane M Griffith, Victor SC Fung. Department of Neurology, Westmead Hospital, Sydney, NSW, Australia

10.1136/jnnp-2019-anzan.31

Introduction We report a prospective, open-label study of 24hour levodopa-carbidopa intestinal gel (LCIG) as treatment for levodopa 'unresponsive' freezing of gait (FOG) associated with Parkinson's disease.

Method 14 patients with disabling FOG, documented as being levodopa 'unresponsive' following dose cycle assessment, were commenced on continuous 24-hour infusion LCIG therapy with the night-time rate at $50-80 \%$ of the daytime infusion rate. Patients underwent baseline and 6 months gait assessments, documentation of their falls frequency and completed FOG questionnaires. The statistical analysis was performed with SPSS 25 software (IBM), using Wilcoxon Signed Rank test and $\mathrm{p}<0.05$ was set as statistically significant.

Result The fall frequency score reduced from 3 to 1 at 6 months $(p=0.011)$, The FOG questionnaire was $19.3 \pm 1.5$ at baseline and reduced to $16.0 \pm 1.7$, at 6 months, respectively $(p=0.039)$. The mean 360 degree turn time was not significantly changed $(p=0.77)$. The daytime LCIG infusion rate was not significantly different before and after 24-hour LCIG therapy $(p=0.315) .7$ subjects developed visual hallucinations, one required temporary withdrawal from 24-hour LCIG, but as FOG worsened on 16-hour LCIG, he elected to recommence 24-hour LCIG infusion with improvement in FOG with a reduction in falls. 1 subject had postural hypotension which was ameliorated after adjustment of LCIG infusion rate.

Conclusion We provide further evidence that 24-hour LCIG therapy can reduce levodopa 'unresponsive' FOG and associated falls.

\section{VALPROATE-INDUCED PARKINSONISM 'AN EARLY WARNING': CASE REPORTS AND REVIEW OF LITERATURE}

${ }^{1}$ Shoaib Dal ${ }^{*},{ }^{1,2}$ Scott Whyte. 'Central Coast Local Health District, Gosford, NSW, Australia; ${ }^{2}$ University of Newcastle, Newcastle, NSW, Australia

10.1136/jnnp-2019-anzan.32

Introduction We describe two cases of valproate-induced parkinsonism, where the parkinsonian features develop after commencing valproate (VPA), in a time frame and manner well described by previous publications. ${ }^{1}$ In contrast to the published literature, that has short duration of follow-up, we have had a prolonged follow-up of these two cases, who initially improved on ceasing valproate, but then after a period progressed again, with the development of levodopa-responsive asymmetrical Parkinson's disease. ${ }^{2}$
Cases 67 years old man, on VPA $500 \mathrm{mg}$ twice a day for seizures since 2008, developed new rest, re-emergent left hand tremor and hypomimia in 2012. Within months, the parkinsonism progressed to decremental bradykinesia, cog-wheel rigidity and hypophonia. The symptoms initially improved with changing VPA to levetiracetam; however, the disease reappeared after 6 months and although levodopa-responsive, it has been progressive since then.

88 years old man, on VPA $700 \mathrm{mg}$ twice a day for seizures since 2010, developed upper limb decremental bradykinesia, cog-wheel rigidity, camptocormia and rest, re-emergent tremor in hands in 2011. The parkinsonism improved significantly with cessation of VPA; however, the disease re-emerged after 2 years and has been progressive since then.

Conclusion VPA may be responsible for unmasking underlying subclinical parkinsonism and these patients represent an earlier onset of Parkinson's disease related to VPA exposure, rather than a purely drug related parkinsonian dysfunction. These cases also highlight the importance of long-term follow up in VPA-induced parkinsonism. These observations are an important consideration for clinicians treating patients with parkinsonian features secondary to VPA exposure.

\section{REFERENCES}

1. Brugger F, Bhatia KP, Besag FM. Valproate-associated parkinsonism: a critical review of the literature. CNS Drugs 2016;30(6):527-540.

2. Mahmoud F, Tampi RR. Valproic acid-induced parkinsonism in the elderly: a comprehensive review of the literature. Am J Geriatr Pharmacother 2011;9 (6):405-412.

\section{THE IMPACT OF A CLINICAL SUPPORT TOOL ON STROKE KEY PERFORMANCE INDICATORS}

Khaled Alanati*, James Evans. Department of Neuroscience, Gosford Hospital, Gosford, NSW, Australia

\subsection{6/jnnp-2019-anzan.33}

Introduction Adherence to key performance indicators (KPIs) in stroke care is associated with better outcomes. ${ }^{1-6}$ The complexity in management of acute strokes, however, has created barriers towards delivering best care with plateauing of KPIs as measured by The National Stroke Foundation Clinical Audit.

We examined the impact on stroke KPIs in our local health district of a web-based decision support stroke platform which provides clinicians with up-to-date information about the patient's management flagging potential areas for improvement, allowing treatment to be optimised in real time.

Methods Six months following the introduction of the platform we performed a retrospective analysis of Electronic medical records of patients admitted to Gosford hospital with acute stroke between June 2018 and September 2018 assessing access to the stroke unit as well as being discharged on appropriate secondary prophylactics, including antihypertensives and correct antithrombotic therapy. Patients whose direction of care was palliative and patients with documented contraindication to secondary prophylactics were excluded.

Results Over four months, 136 patients presented with acute ischaemic stroke and 11 patients had a haemorrhagic stroke. 49 ischaemic stroke patients had atrial fibrillation. Stroke unit access was higher following its introduction in 2018 compared 
to 2017 (97\% vs 76\%, respectively). Similar findings were noted for patients with atrial fibrillation who received oral anticoagulants on discharge $(90 \%$ vs $50 \%)$ and patients discharged on antihypertensives (95\% vs $80 \%$ ).

Conclusion Use of a clinical support platform in managing acute stroke is an intervention that improves stroke care.

\section{REFERENCES}

1. Urimubenshi G, Langhorne P, Cadilhac DA, Kagwiza JN, Wu O. Association between patient outcomes and key performance indicators of stroke care quality: A systematic review and meta-analysis. European Stroke Journal 2017;2(4):287307. https://doi.org/10.1177/2396987317735426

2. Sandercock P, Gubitz G, Foley P. Antiplatelet therapy for acute ischaemic stroke. Cochrane Database Syst Rev 2003;2: CD000029. Google Scholar

3. Kwan J, Sandercock P. In-hospital care pathways for stroke. Cochrane Database Syst Rev 2004;4: CD002924. Google Scholar

4. Saxena R, Koudstaal PJ. Anticoagulants for preventing stroke in patients with nonrheumatic atrial fibrillation and a history of stroke or transient ischemic attack. Cochrane Database Syst Rev 2004;4: CD000187. Google Scholar

5. Goyal M, Menon BK, van Zwam WH. Endovascular thrombectomy after large-vessel ischaemic stroke: a meta-analysis of individual patient data from five randomised trial. Lancet 2016;387:1723-1731. Google Scholar | Crossref | Medline | $|S|$

6. Middleton $S$, McElduff $P$, Ward J. Implementation of evidence-based treatment protocols to manage fever, hyperglycaemia, and swallowing dysfunction in acute stroke (QASC): a cluster randomised controlled trial. Lancet 2011;378:16991706. Google Scholar | Crossref | Medline | IS|

\section{FRONTOTEMPORAL DEMENTIA OR FRONTAL VARIANT ALZHEIMER'S DISEASE? A CASE SERIES}

${ }^{1,2}$ Alice Powell ${ }^{*},{ }^{3}$ David Foxe, ${ }^{3}$ Glenda M Halliday, ${ }^{3}$ Olivier Piguet, ${ }^{3}$ John R Hodges,

1,3 James R Burrell. ' Concord Repatriation General Hospital, Sydney, NSW, Australia; ${ }^{2}$ Brain and Mind Centre, University of Sydney, Redfern, NSW, Australia; ${ }^{3}$ Brain and Mind Centre, The University of Sydney, Sydney, NSW, Australia

10.1136/jnnp-2019-anzan.34

Introduction Accurate prediction of the underlying neuropathology in behavioural variant frontotemporal dementia (bvFTD) is essential for future targeted therapy trials and prognostication. Alzheimer's disease (AD) pathology has been reported in a significant proportion of patients with clinical bvFTD. We sought to determine whether detailed clinical and neuroradiological assessment was sufficient to distinguish bvFTD with AD pathology from bvFTD with frontotemporal lobar degeneration (FTLD).

Methods Two patients with clinically diagnosed probable bvFTD but AD pathology at autopsy, were identified. The clinical, neuropsychological and imaging features of these patients were compared with those of ten patients with clinically probable bvFTD and proven FTLD pathology (tau, TDP43, FUS).

Results Both patients with AD pathology presented with behavioural symptoms typical of bvFTD as well as memory impairment. Executive function, memory and visuospatial skills were impaired in both pathologic groups. Language skills were relatively spared in those with $\mathrm{AD}$ pathology. Neuropsychiatric symptoms were frequent in both groups but significant depression and anxiety were seen only in those with FTLD pathology. Dementia severity and caregiver burden were similar. The degree or topographical distribution of atrophy on MRI did not differ.

Conclusions Alzheimer's pathology may cause bvFTD symptoms which are otherwise indistinguishable to those caused by FTLD pathology. While there may be subtle differences in patterns of cognitive deficits, standard neuropsychological testing is insufficient to discern the underlying pathology.
Similarly, structural imaging cannot be used to reliably identify $\mathrm{AD}$ pathology. Better access to amyloid biomarkers may be needed to more accurately define bvFTD caused by $A D$ pathology.

\section{TREMOR: A CLINICAL AND NEUROPHYSIOLOGICAL STUDY}

${ }^{2,1}$ Alessandro Fois ${ }^{*},{ }^{2,1}$ Neil Mahant, ${ }^{2,1}$ Steve Vucic, ${ }^{2,1}$ Victor SC Fung. ${ }^{1}$ Movement Disorders Unit, Westmead Hospital, Westmead, NSW, Australia; '2Sydney Medical School, the University of Sydney, Sydney, NSW, Australia

\subsection{6/jnnp-2019-anzan.35}

Introduction Tremor is a common clinical problem seen in a number of diseases. Robust classification and diagnosis of tremor remains controversial due to overlap in clinical features and lack of established biomarkers. This hampers effective research including therapeutic trials. We present our research protocol for a cross-sectional study which aims to find more robust methods of tremor classification and diagnosis.

Methods Adults with upper limb tremor of varying aetiologies, diagnosed using current clinical criteria (including essential tremor, Parkinsonian tremor, and dystonic tremor), and age-matched controls are eligible for recruitment. Participants undergo a clinical and neurophysiological assessment, including accelerometry, surface electromyography, long-latency stretch reflexes, temporal discrimination, and tonic vibration reflexes. Data will be analysed using a cluster analysis to identify robust tremor syndromes and biomarkers associated with them. We aim to recruit 100 participants prior to analysis.

Results At time of writing, 13 participants with upper limb tremor have been studied (6 with essential tremor, 5 with dystonic tremor, and 2 with indeterminate tremor; mean age 66 years, range 18-85). Participants tolerated the clinical and neurophysiological studies well with $100 \%$ completion rate after recruitment. With current rates of recruitment we anticipate completion of recruitment and commencement of data analysis in October 2019.

Conclusions Our protocol aims to identify robust tremor phenotypes and biomarkers for them. This will allow patients with tremor to be classified into more biologically homogeneous diagnostic categories, aiding future research into the mechanism of tremor and more rational clinical trial design.

\section{ESTIMATING THE HEALTH AND ECONOMIC BURDEN OF INVESTIGATING TUMEFACTIVE DEMYELINATION COMPARED TO CONVENTIONAL MULTIPLE SCLEROSIS}

${ }^{1}$ Matthew Silsby*, ${ }^{2}$ Pedro S Lopez, ${ }^{3,2}$ Judy Spies, ${ }^{1,2}$ Stephen Reddel, ${ }^{2}$ Jane Frith, ${ }^{2}$ Joshua Barton, ${ }^{3,2}$ Heidi Beadnall, ${ }^{3,2}$ Michael Barnett, ${ }^{1,2}$ Todd Hardy. ${ }^{1}$ Neurology, Concord Hospital, Concord, NSW, Australia; '2Sydney Neurology, Brain and Mind Centre, Camperdown, NSW, Australia; ${ }^{3}$ Neurology, Royal Prince Alfred Hospital, Sydney, NSW, Australia

\subsection{6/jnnp-2019-anzan.36}

Introduction Tumefactive demyelinating lesions, defined as demyelinating lesions $>2 \mathrm{~cm}$ in diameter, occur most commonly in association with multiple sclerosis (MS), and can pose a diagnostic challenge. The aim of this study was to 